\title{
Interstitial 4q Deletion Syndrome Including NR3C2 Causing Pseudohypoaldosteronism
}

\author{
Amanda Barone Pritchard ${ }^{a, b} \quad$ Alyssa Ritter $^{a} \quad$ Hutton M. Kearney ${ }^{c}$ \\ Kosuke Izumi ${ }^{a}$ \\ a Division of Human Genetics, Department of Pediatrics, The Children's Hospital of Philadelphia, Philadelphia, PA, \\ USA; ${ }^{b}$ Division of Pediatric Genetics, Metabolism, and Genomic Medicine, Department of Pediatrics, University of \\ Michigan, Ann Arbor, MI, USA; 'Division of Laboratory Genetics and Genomics, Mayo Clinic, Rochester, MN, USA
}

\section{Established Facts}

- Interstitial and terminal deletions of chromosome 4q have variable phenotypes including developmental delay, growth difficulty, digital differences, dysmorphic features, and cardiac anomalies.

- Mutations in the gene NR3C2, located in chromosome 4q, cause autosomal dominant pseudohypoaldosteronism type 1 (PHA1A).

\section{Novel Insights}

- This case is a patient with a rare 6.4-Mb deletion of $4 \mathrm{q} 31.21 \mathrm{q} 31.23$ found to have PHA1A due to deletion of NR3C2.

- Patients with $4 \mathrm{q}$ deletions including NR3C2 should be evaluated for PHA1A, as this can at times be subtle or minimally symptomatic.

\section{Keywords}

Chromosomal microarray · Deletion syndrome - NR3C2 ·

Pseudohypoaldosteronism · $4 q$ - syndrome

\section{Abstract \\ Interstitial and terminal deletions of chromosome $4 q$ have been described for many years and have variable pheno- types depending on the size of the deletion present. Clinical features can include developmental delay, growth difficulty, digital differences, dysmorphic features, and cardiac anoma- lies. Here, we present an infant with pseudohypoaldosteron- ism found to have a deletion of $4 q 31.21 q 31.23$, including}

$N R 3 C 2$. Heterozygous mutations in NR3C2 have been reported to cause autosomal dominant pseudohypoaldosteronism type 1 (PHA1A). This represents a rare case of PHA1 A due to a contiguous interstitial deletion and highlights the importance of evaluating patients with overlapping deletions for PHA1A.

(c) 2019 S. Karger AG, Basel

There have been many cases reported in the literature of $4 \mathrm{q}$ deletion syndrome, also known as $4 \mathrm{q}$ - syndrome, a variable phenotype which can include growth failure, digital anomalies, developmental delay, autism spectrum disorder, cardiac defects, and skeletal defects [Strehle et

\section{KARGER}

(c) 2019 S. Karger AG, Basel

E-Mail karger@karger.com

www.karger.com/msy
Amanda Barone Pritchard, MD

Division of Pediatric Genetics, Metabolism, and Genomic Medicine Department of Pediatrics

C.S. Mott Children's Hospital, Michigan Medicine, D5240 Medical Professional Building 1500 E Medical Center Drive, Ann Arbor, MI 48109 (USA)

E-Mail abarone@med.umich.edu 
al., 2012b]. The incidence has been estimated to be 1 in 100,000 with the majority of deletions occurring de novo [Strehle et al., 2001; Strehle and Bantock, 2003]. The sizes of these deletions and the exact breakpoints vary, ranging from small interstitial deletions to larger terminal deletions. Here, we report a neonate found to have a $6.4-\mathrm{Mb}$ deletion of 4q31.21q31.23 including NR3C2 (OMIM 600983), diagnosed in the setting of pseudohypoaldosteronism (PHA). Overlapping deletions in the literature are rare; of the 6 cases with overlapping deletions, only 4 include $4 \mathrm{q} 31.23 ; 2$ cases were identified by karyotype prior to the availability of microarray [Sarda et al., 1992; Robertson et al., 1998; de Ravel et al., 2009; Strehle et al., 2012b; Duga et al., 2014; Vlaikou et al., 2014]. NR3C2 encodes the mineralocorticoid receptor, which responds to aldosterone to regulate transcription of several target genes to promote fluid balance and sodium and potassium homeostasis [O'Connell et al., 2011]. Heterozygous loss-of-function pathogenic variants in NR3C2 have been shown to cause autosomal dominant pseudohypoaldosteronism type 1 (PHA1A; OMIM 177735) [Geller et al., 2006]. Interstitial $4 \mathrm{q}$ deletion syndrome has only rarely been associated with PHA1A, and we report this uncommon situation and discuss clinical implications.

\section{Clinical Report and Methods}

The patient was first evaluated by genetics as an inpatient at 2 months of age due to an abnormal microarray result. The Caucasian male was born to a 32-year-old primigravid mother by cesarean section at 28 weeks gestation because of maternal pre-eclampsia. The pregnancy was conceived with assistance of clomiphene and intrauterine insemination due to maternal history of polycystic ovarian syndrome. Prenatal ultrasounds were normal with the exception of a 2-vessel umbilical cord. Family history was notable for the father having a history of speech delay; otherwise family history was unremarkable. There was no consanguinity.

At birth, growth parameters for gestational age included weight of 1,425 g (95th percentile, $Z$-score 1.6), length of $40.6 \mathrm{~cm}$ (96th percentile, $Z$-score 1.7), and head circumference of $28 \mathrm{~cm}$ (96th percentile, $Z$-score 1.7). The neonate was transferred to the neonatal intensive care unit due to prematurity. He was found to have bilateral inguinal hernias. While in this unit, the infant had significant electrolyte abnormalities including hyponatremia, hyperkalemia, hypercalcemia, and metabolic acidosis. His electrolyte abnormalities and elevated aldosterone level were most consistent with PHA. A renal and bladder ultrasound was normal, and the infant underwent inguinal hernia repair. Electrolyte abnormalities improved over time with medical management (sodium chloride supplementation and fludrocortisone), and the infant was discharged home on day 75 of life.

Given the diagnosis of PHA, a gene panel including sequencing and deletion/duplication analysis of SCNN1A, SCNN1B, SCNN1G, and NR3C2 was performed. Methods included DNA extraction fol- lowed by next-generation sequencing on the Illumina MiSeq instrument, aligned to human reference genome (GRCh37/hg19) using the Burrows-Wheeler Aligner. Dosage analysis was carried out by normalization of next-generation sequencing read depth. This detected a heterozygous pathogenic whole-gene deletion of NR3C2 (breakpoints could not be determined, reported as c.1-?_2967?del).

A chromosomal microarray was performed using copy number and single-nucleotide polymorphism probes on a whole-genome array with the Affymetrix CytoScan HD platform. Data were analyzed and reported using the February 2009 NCBI human genome build 37.1 (hg19). Based on array results, metaphase and interphase FISH of the region 4q31.22 was performed using the CTD2228015 probe (Mayo Clinic, Laboratory Developed Test) in the patient and his parents.

The infant returned to the genetics clinic at 9.75 months of age ( 6.75 months corrected given prematurity). He required nasogastric tube feeds until approximately 5 months of age. The infant had mild developmental delay; he was reaching for objects by 2 months of age and rolled over from front to back inconsistently at 9 months of age. At 9 months of age, he was not yet sitting unsupported. He received therapies through Early Intervention including physical therapy and occupational therapy. Physical examination at that time was notable for sparse eyebrows, normal eyelids, a bifid nasal tip with upturned nose, normal appearance of ears, sparse scalp hair, and bilateral 5th finger clinodactyly. Growth parameters (corrected for prematurity) included weight of $6.44 \mathrm{~kg}$ (1st percentile, $Z$-score -2.2), length of $64.8 \mathrm{~cm}$ (8th percentile, $Z$-score -1.4$)$, and head circumference of $41.7 \mathrm{~cm}$ (3rd percentile, $Z$-score -1.9). In terms of management of his PHA, fludrocortisone was discontinued at 5 months of age, and he remained on sodium chloride supplements.

\section{Results}

The chromosomal microarray revealed an approximately 6.4-Mb deletion from $4 \mathrm{q} 31.21$ to $4 \mathrm{q} 31.23$ (arr[GRCh37] 4q31.21q31.23(143413519_149801344)×1), including NR3C2 (Fig. 1), in the patient. No additional pathologic copy number variants were identified. This deletion was also confirmed by FISH, ish 4q31.22(CTD-2228O15×1). Both parents showed a normal FISH pattern, ish $4 q 31.22$ (CTD-2228O15×2), confirming a de novo origin in our patient. No parental rearrangements were identified.

\section{Discussion}

We present a rare case of $4 \mathrm{q}$ interstitial deletion syndrome with associated PHA. PHA1A is known to be caused by heterozygous mutations or disruptions in NR3C2 and is inherited in an autosomal dominant manner [Pujo et al., 2007; O'Connell et al., 2011; Casas-Alba et al., 2017]. Some individuals may be only subclinically affected with PHA, and even those that are affected tend to have a mild course and outgrow the need for treatment 


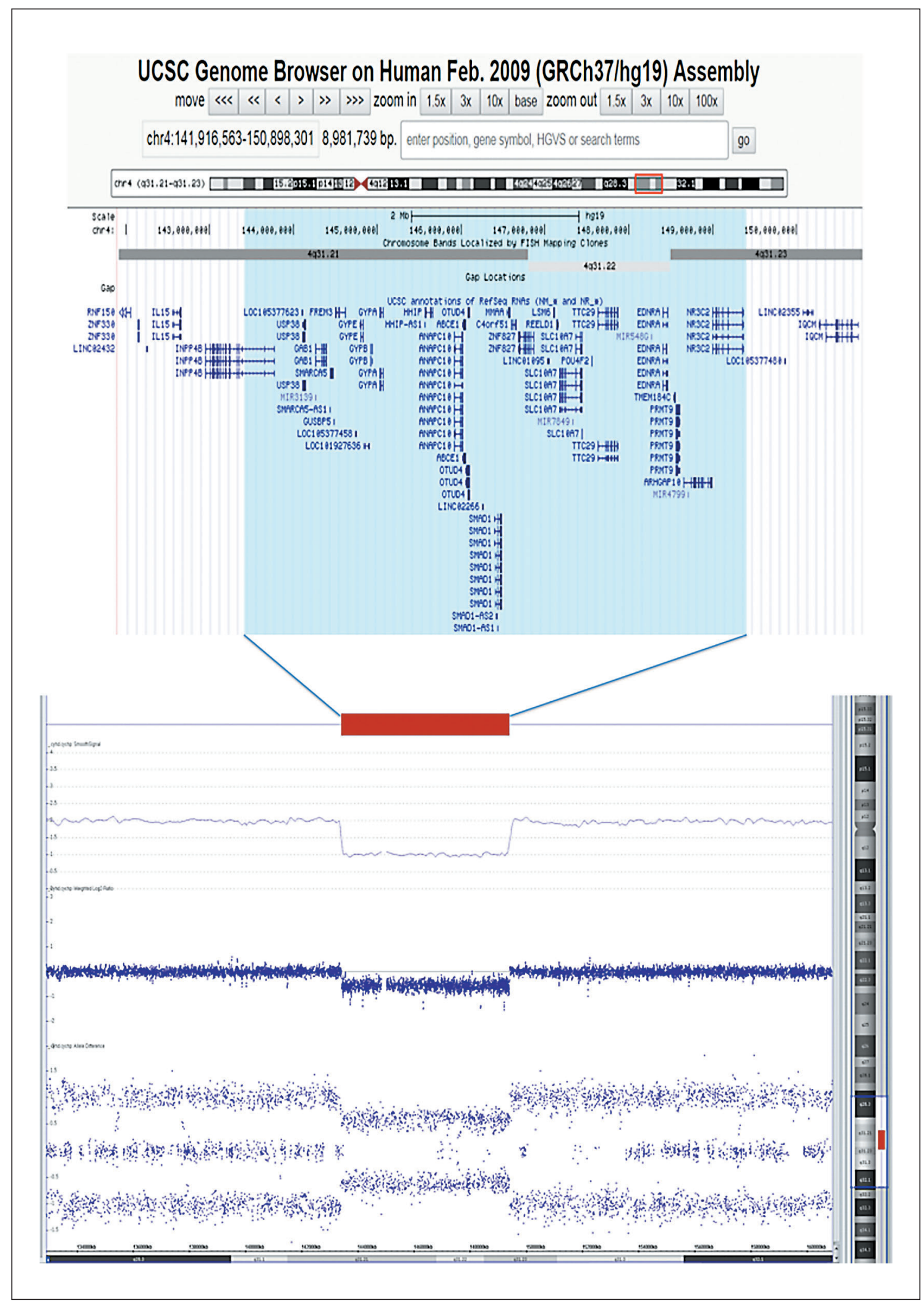

Fig. 1. UCSC Genome Browser [Kent et al., 2002] view of the deletion. The shaded region shows the deleted genes. A microarray data plot is located below, with lines indicating the overlapping region in UCSC Genome Browser. 
by several years of age. Large deletions including single or multiple exons of NR3C2 have also been observed in patients with PHA1A [Pujo et al., 2007].

In the literature, individuals with overlapping deletions in the $4 \mathrm{q}$ region have been reported to have speech delay, learning difficulties, macrocephaly, frontal bossing, prominent upper lip, bilateral postaxial polydactyly, and seizures [Strehle and Bantock, 2003; Strehle et al., 2012a; Vlaikou et al., 2014]. While many 4q- patients are described in the literature, only 2 of these had features that could be consistent with PHA [de Ravel et al., 2009; Duga et al., 2014]. Variable penetrance of PHA has been noted, as some individuals with NR3C2 mutations were unaffected or had a mild phenotype that was unrecognized [Riepe et al., 2006]. Functional studies indicated that mutations in NR3C2 cause disease via haploinsufficiency [Geller et al., 2006]; therefore, it is not surprising that the wholegene deletion in this case resulted in the clinical phenotype of PHA1A. A previously reported patient with PHA1A was found to have an intragenic deletion of exons 3-5 of NR3C2 identified on microarray, providing further support for loss-of-function through heterozygous deletion resulting in PHA1A [Bowden et al., 2013].

Our patient shares several features described previously in patients with overlapping $4 \mathrm{q}$ deletions including developmental delay, digital abnormalities, and differences in nose shape. While PHA1A has not been reported in the majority of patients, it is certainly possible that subclinical cases were missed. Two cases of deletions including 4q31.23 identified by karyotype have no mention of electrolyte abnormalities; however, microarray data are not available to confirm the breakpoints of the deletions and inclusion (or exclusion) of NR3C2 [Sarda et al., 1992; Robertson et al., 1998]. One case diagnosed with Opitz C trigonocephaly syndrome and PHA published in 1990 was later determined to harbor a microdeletion of $4 \mathrm{q} 28.3 \mathrm{q} 31.23$, resulting in a proximal deletion of NR3C2 [de Ravel et al., 2009]. There is also a case report of a patient with a 4q28.3q31.23 deletion including NR3C2 who was noted to have hyponatremia; this infant passed away due to other comorbidities [Duga et al., 2014]. The authors proposed the hyponatremia may have been caused by the deletion of NR3C2 but did not specifically link this to PHA1A. It is highly possible that the hyponatremia in this case was related to unrecognized PHA. Hence, close monitoring of sodium level in individuals with 4q deletion including NR3C2 is strongly recommended.

The deletion in our case includes 37 genes, most of which are currently not associated with a recognized clinical phenotype. Genes known to be linked with autosomal recessive conditions in this region include GAB1 (OMIM 604439; autosomal recessive deafness-26), SLC10A7 (OMIM 611459; short stature, amelogenesis imperfecta, and skeletal dysplasia with scoliosis), and MMAA (OMIM 607481; vitamin B12-responsive methylmalonic aciduria). The infant had a normal hearing screen, a normal metabolic newborn screen, and no evidence of skeletal dysplasia; so it is unlikely that he harbors a mutation in one of these genes on the other allele. The only gene known to be associated with an autosomal dominant condition in this region aside from NR3C2 is EDNRA (OMIM 131243). Specific pathogenic gain-of-function variants of EDNRA are associated with autosomal dominant mandibulofacial dysostosis with alopecia, which does neither fit this patient's phenotype nor mutational mechanism [Gordon et al., 2015].

Our case of $4 \mathrm{q}-$ syndrome was diagnosed due to his symptomatic $\mathrm{PHA}$, but 4q microdeletion syndrome may be identified by other clinical features in patients without overt symptoms of PHA. Any patient with a 4q deletion including NR3C2 thus warrants endocrinologic evaluation given the variable symptoms of PHA. Additionally, any gene panel revealing a large deletion of $N R 3 C 2$ should prompt a microarray to evaluate for larger contiguous deletions. It is certainly feasible that mild cases of PHA1A have been missed in other $4 \mathrm{q}$ deletion patients. More rigorous study is needed to determine the frequency of clinically relevant PHA in patients found to have deletions of NR3C2.

\section{Acknowledgements}

The authors would like to thank the patient's family for allowing us to share this case.

\section{Statement of Ethics}

The parents of the patient have given written informed consent for publication of this case.

\section{Disclosure Statement}

The authors have no conflicts of interest to declare.

\section{Author Contributions}

All authors were involved in conception and design of the work. A.B.P. wrote the initial draft, which underwent critical revisions by K.I. and A.R. H.M.K. provided the figure and additional critical revisions. The authors are accountable for this work and have approved it for publication. 


\section{References}

Bowden SA, Cozzi C, Hickey SE, Thrush DL, Astbury C, Nuthakki S: Autosomal dominant pseudohypoaldosteronism type 1 in an infant with salt wasting crisis associated with urinary tract infection and obstructive uropathy. Case Rep Endocrinol 2013:524647 (2013).

Casas-Alba D, Vila Cots J, Monfort Carretero L, Martorell Sampol L, Zennaro MC, et al: Pseudohypoaldosteronism types I and II: little more than a name in common. J Pediatr Endocrinol Metab 30:597-601 (2017).

$\checkmark$ de Ravel T, Balikova I, Van Driessche J, Vermeesch J, Fryns JP: "Opitz C syndrome and pseudohypoaldosteronism" is caused by a chromosome 4q deletion. Am J Med Genet A 149A:1315-1316 (2009).

Duga B, Czako M, Komlosi K, Hadzsiev K, Torok $\mathrm{K}$, et al: Deletion of 4q28.3-31.23 in the background of multiple malformations with pulmonary hypertension. Mol Cytogenet 7:36 (2014).

-Geller DS, Zhang J, Zennaro MC, Vallo-Boado A, Rodriguez-Soriano J, et al: Autosomal dominant pseudohypoaldosteronism type 1 : mechanisms, evidence for neonatal lethality, and phenotypic expression in adults. J Am Soc Nephrol 17:1429-1436 (2006).
Gordon CT, Weaver KN, Zechi-Ceide RM, Madsen EC, Tavares AL, et al: Mutations in the endothelin receptor type A cause mandibulofacial dysostosis with alopecia. Am J Hum Genet 96:519-531 (2015).

Kent WJ, Sugnet CW, Furey TS, Roskin KM, Pringle TH, et al: The human genome browser at UCSC. Genome Res 12:996-1006 (2002).

O'Connell SM, Johnson SR, Lewis BD, Staltari L, Peverall J, et al: Structural chromosome disruption of the NR3C2 gene causing pseudohypoaldosteronism type 1 presenting in in fancy. J Pediatr Endocrinol Metab 24:555559 (2011).

Pujo L, Fagart J, Gary F, Papadimitriou DT, Claes A, et al: Mineralocorticoid receptor mutations are the principal cause of renal type 1 pseudohypoaldosteronism. Hum Mutat 28: 33-40 (2007)

Riepe FG, Finkeldei J, de Sanctis L, Einaudi S, Testa A, et al: Elucidating the underlying molecular pathogenesis of NR3C2 mutants causing autosomal dominant pseudohypoaldosteronism type 1. J Clin Endocrinol Metab 91:45524561 (2006).
Robertson SP, O’Day K, Bankier A: The 4q-syndrome: delineation of the minimal critical region to within band 4q31. Clin Genet 53:7073 (1998).

- Sarda P, Lefort G, Fryns JP, Humeau C, Rieu D: Interstitial deletion of the distal long arm of chromosome 4. J Med Genet 29:259-261 (1992).

Strehle EM, Bantock HM: The phenotype of patients with 4q-syndrome. Genet Couns 14: 195-205 (2003).

Strehle EM, Ahmed OA, Hameed M, Russell A: The 4q-syndrome. Genet Couns 12:327-339 (2001).

Strehle EM, Gruszfeld D, Schenk D, Mehta SG, Simonic I, Huang T: The spectrum of $4 \mathrm{q}-$ syndrome illustrated by a case series. Gene 506:387-391 (2012a).

Strehle EM, Yu L, Rosenfeld JA, Donkervoort S Zhou Y, et al: Genotype-phenotype analysis of $4 \mathrm{q}$ deletion syndrome: proposal of a critical region. Am J Med Genet A 158A:2139-2151 (2012b).

Vlaikou AM, Manolakos E, Noutsopoulos D, Markopoulos G, Liehr T, et al: An interstitial $4 \mathrm{q} 31.21 \mathrm{q} 31.22$ microdeletion associated with developmental delay: case report and literature review. Cytogenet Genome Res 142:227238 (2014). 4q Deletion Including NR3C2 with Pseudohypoaldosteronism 